\title{
STATIC SUSCEPTIBILITIES OF NONUNIFORM AND RANDOM ISING CHAINS
}

\author{
O. Derzhko, O. Zaburannyi \\ Institute for Condensed Matter Physics of the National Academy of Sciences of Ukraine \\ 1 Svientsitskii Str., Lviv, UA-290011, Ukraine \\ Tel: (0322)427439,fax: (0322)761978,e-mail: derzhko@icmp.lviv.ua \\ Ivan Franko Lviv State University, Chair of Theoretical Physics \\ 12 Drahomanov Str., Lviv, UA-290005, Ukraine
}

(Received June 4, 1997)

\begin{abstract}
We have derived exact results for the initial static susceptibilities of nonuniform spin- $\frac{1}{2}$ Ising chains. The results obtained permit one to study the random-bond Ising chain. The influence of several types of disorder on the temperature dependence of the initial static susceptibilities is discussed
\end{abstract}

Key words: random-bond Ising chain, susceptibility.

PACS number(s): 75.10.-b

\section{INTRODUCTORY REMARKS}

The model formulated by Ising in 1925 [1] is still widely used in condensed matter theory as a laboratory for performing statistical mechanics calculations without making any approximations. Such exact results provide an intuition that is important in more realistic cases and permits one to test approximate approaches as well as allows one to suggest new approximations.

The present paper deals with the initial (zero-field) static susceptibilities of nonuniform and random spin- $\frac{1}{2}$ Ising chains. The spin model considered is governed by the Hamiltonian

$$
H_{0}=\sum_{n} J_{n} s_{n}^{x} s_{n+1}^{x}
$$

Here (and throughout the paper) the summation is performed over $N$ lattice sites, $J_{n}$ is the exchange coupling between the $n$ and $n+1$ sites, and $s^{x}$ is a half of the Pauli matrix $\left(\begin{array}{cc}1 & 0 \\ 0 & -1\end{array}\right)$. The boundary conditions are not written down explicitly. We shall use both cyclic and open boundary conditions depending on calculational convenience. For deriving the static susceptibilities we shall switch on a small field $h_{\alpha}, \alpha=x, y, z$, so that the total Hamiltonian will be

$$
H=H_{0}+H_{\alpha}, \quad H_{\alpha}=h_{\alpha} \sum_{n} s_{n}^{\alpha}
$$

Since susceptibility measurements are one of the basic experimental techniques in the study of magnetic compounds, the importance of having accurate results for the initial static susceptibilities is clear.

Let us at first recall some well-known results. In the case of the uniform Ising model in a longitudinal field $\left(J_{n}=J, \alpha=x\right)$ the Helmholtz free energy per site $f\left(T, h_{x}\right)$ may be found with the help of the transfer matrix method [2]. Differentiating this expression twice with respect to $h_{x}$ one gets the longitudinal static susceptibility

$$
\chi_{x x}=-\frac{1}{4 k T} \frac{\mathrm{e}^{\frac{J}{4 k T}} \cosh \frac{h_{x}}{2 k T}}{\left(\mathrm{e}^{-\frac{J}{2 k T}} \sinh ^{2} \frac{h_{x}}{2 k T}+\mathrm{e}^{\frac{J}{2 k T}}\right)^{\frac{3}{2}}} .
$$

In the case of the uniform Ising model in a transverse field $\left(J_{n}=J, \alpha=z\right)$ one may use the method developed by Lieb, Schultz and Mattis [3,4]. After Jordan-Wigner transformation the spin Hamiltonian exhibits a bilinear Fermi-form that can be diagonalized, and which in turn yields immediately the Helmholtz free energy per site $f\left(T, h_{z}\right)$. After differentiating $f\left(T, h_{z}\right)$ twice with respect to $h_{z}$ one gets the transverse static susceptibility

$$
\begin{aligned}
& \chi_{z z}=-\frac{1}{4 k T}\left[\frac{1}{2 \pi} \int_{-\pi}^{\pi} d \kappa \frac{1}{\cosh ^{2} \frac{\Lambda_{\kappa}}{2 k T}} \frac{\left(h_{z}+\frac{J}{2} \cos \kappa\right)^{2}}{\Lambda_{\kappa}^{2}}+\frac{k T}{\pi} \int_{-\pi}^{\pi} d \kappa \tanh \frac{\Lambda_{\kappa}}{2 k T} \frac{\frac{J^{2}}{4} \sin ^{2} \kappa}{\Lambda_{\kappa}^{3}}\right] \\
& \Lambda_{\kappa}=\sqrt{h_{z}^{2}+h_{z} J \cos \kappa+\frac{J^{2}}{4}} .
\end{aligned}
$$


We should also mention here the related study of the zero-temperature susceptibilities of the Ising chain in a transverse field obtained on the basis of a rigorous numerical approach for the calculation of dynamic spin correlation functions $[5,6]$.

Since the 60 s there has been considerable interest in disordered versions of the Ising chain, but the results achieved are not so impressive as for the nonrandom case. In ref. [7] the Ising chain with random exchange energy in a longitudinal field was considered. Assuming that each exchange interaction was an independent random variable having a probability density function with a narrow width, Fan and McCoy were able to study the influence of the randomness on the longitudinal static susceptibility. The ground-state transverse static susceptibility for the random-bond Ising chain was examined by Barouch and McCoy [8]. Zaitsev studied the transverse static susceptibility for the random-bond Ising chain for various types of disorder [9]. Thermodynamics and spin correlations of a model of solid solution based on Ising chain were considered in ref. [10]. Some results for the longitudinal and transverse initial static susceptibilities of the random Ising chain in a transverse field are collected in ref. [11]. Thermodynamic and dynamic properties of a random Ising chain in a transverse field for arbitrary disorder may be also examined numerically [12]. We should also mention here the related problem of the calculation of the spin correlation functions for random Ising chains (see, for example, ref. [13]).

Recently, Idogaki, Rikitoku and Tucker [14] calculated exactly the initial transverse static susceptibility of nonuniform and random Ising chains by a method based on the use of the Callen-Suzuki identity. In addition, they studied the random chain having two types of exchange bonds. The goal of the present paper is twofold. First, we shall present what, in our opininon, is a more simple derivation of the initial transverse static susceptibility of a nonuniform Ising chain, and consider in addition the other components of the susceptibility tensor (Section II). Secondly, we shall proceed to study random Ising chains, considering in particular, some other types of disorder (Section III). The exact calculations presented are important for understanding the influence of disorder on the temperature dependence of the initial static susceptibilities of Ising chains.

\section{INITIAL STATIC SUSCEPTIBILITIES OF A NONUNIFORM ISING CHAIN}

We consider the system described by the Hamiltonian (2) which exhibits the magnetization $m_{\beta} \equiv$ $\frac{1}{N} \sum_{n}<s_{n}^{\beta}>$. We are interested in the evaluation of the static susceptibility $\chi_{\beta \alpha} \equiv \frac{\partial m_{\beta}}{\partial h_{\alpha}}$. It is generally known that

$$
\chi_{\beta \alpha}=-\frac{1}{k T} \frac{1}{N} \sum_{n, p}\left[\int_{0}^{1} d \tau<s_{n}^{\beta}\left(-\frac{i \tau}{k T}\right) s_{p}^{\alpha}>-<s_{n}^{\beta}><s_{p}^{\alpha}>\right]
$$

(see for instance ref. [15]). Here the thermal average is defined as $<(\ldots)>\equiv \operatorname{Tr}\left[\mathrm{e}^{-\frac{H}{k T}}(\ldots)\right] / \operatorname{Tr} \mathrm{e}^{-\frac{H}{k T}}$, and $s_{n}^{\beta}(t) \equiv$ $\mathrm{e}^{i t H} s_{n}^{\beta} \mathrm{e}^{-i t H}$. In particular, from eq. (5) it follows that

$$
\chi_{x x}=-\frac{1}{k T} \frac{1}{N} \sum_{n, p}\left(<s_{n}^{x} s_{p}^{x}>-<s_{n}^{x}><s_{p}^{x}>\right)
$$

and

$$
\chi_{z z}=-\frac{1}{k T} \frac{1}{N} \sum_{n, p}\left[\int_{0}^{1} d \tau<s_{n}^{z}\left(-\frac{i \tau}{k T}\right) s_{p}^{z}>-<s_{n}^{z}><s_{p}^{z}>\right]
$$

As we are focusing attention on the initial susceptibilities, when $h_{\alpha}=0$, the thermal average is performed with the Hamiltonian $H_{0}(1)$; such an average will be denoted as $<(\ldots)>_{0}$. Then the expressions for $\chi_{x x}$ and $\chi_{z z}$ simplify since $<s_{n}^{\alpha}>_{0}=0$ (see below).

The next step is to do the integral in eq. (7). Using the identity $\mathrm{e}^{\lambda s^{x}}=\cosh \frac{\lambda}{2}+2 \sinh \frac{\lambda}{2} s^{x}$, one finds that

$$
s_{j}^{z}(t)=\cos \left[t\left(J_{j-1} s_{j-1}^{x}+J_{j} s_{j+1}^{x}\right)\right] s_{j}^{z}+\sin \left[t\left(J_{j-1} s_{j-1}^{x}+J_{j} s_{j+1}^{x}\right)\right] s_{j}^{y},
$$

which since $\cos \left(\lambda s^{x}\right)=\cos \frac{\lambda}{2}, \quad \sin \left(\lambda s^{x}\right)=2 \sin \frac{\lambda}{2} s^{x}$, becomes 


$$
\begin{aligned}
s_{j}^{z}(t) & =\left(\cos \frac{t J_{j-1}}{2} \cos \frac{t J_{j}}{2}-4 \sin \frac{t J_{j-1}}{2} \sin \frac{t J_{j}}{2} s_{j-1}^{x} s_{j+1}^{x}\right) s_{j}^{z} \\
& +2\left(\sin \frac{t J_{j-1}}{2} \cos \frac{t J_{j}}{2} s_{j-1}^{x}+\cos \frac{t J_{j-1}}{2} \sin \frac{t J_{j}}{2} s_{j+1}^{x}\right) s_{j}^{y} .
\end{aligned}
$$

The integration in eq. (7) then yields the desired result

$$
\begin{aligned}
\chi_{z z}= & -\frac{1}{N} \sum_{n, p}\left[\left(\frac{\sinh \frac{J_{n-1}-J_{n}}{2 k T}}{J_{n-1}-J_{n}}+\frac{\sinh \frac{J_{n-1}+J_{n}}{2 k T}}{J_{n-1}+J_{n}}\right)<s_{n}^{z} s_{p}^{z}>_{0}\right. \\
& -4\left(\frac{\sinh \frac{J_{n-1}-J_{n}}{2 k T}}{J_{n-1}-J_{n}}-\frac{\sinh \frac{J_{n-1}+J_{n}}{2 k T}}{J_{n-1}+J_{n}}\right)<s_{n-1}^{x} s_{n}^{z} s_{n+1}^{x} s_{p}^{z}>_{0} \\
& -2 i\left(\frac{\cosh \frac{J_{n-1}-J_{n}}{2 k T}-1}{J_{n-1}-J_{n}}+\frac{\cosh \frac{J_{n-1}+J_{n}}{2 k T}-1}{J_{n-1}+J_{n}}\right)<s_{n-1}^{x} s_{n}^{y} s_{p}^{z}>_{0} \\
& \left.+2 i\left(\frac{\cosh \frac{J_{n-1}-J_{n}}{2 k T}-1}{J_{n-1}-J_{n}}-\frac{\cosh \frac{J_{n-1}+J_{n}}{2 k T}-1}{J_{n-1}+J_{n}}\right)<s_{n}^{y} s_{n+1}^{x} s_{p}^{z}>_{0}\right] .
\end{aligned}
$$

One now faces the problem of calculating the product of several spin operators for a nonuniform Ising chain. However the operators $s^{y}$ and $s^{z}$ rotate the spin, whereas $s^{x}$ does not. Therefore, $<s_{n}^{z}>_{0}=0,<s_{n}^{z} s_{p}^{z}>_{0}=$ $\frac{1}{4} \delta_{n p},<s_{n-1}^{x} s_{n}^{z} s_{n+1}^{x} s_{p}^{z}>_{0}=\frac{1}{4} \delta_{n p}<s_{n-1}^{x} s_{n+1}^{x}>_{0},<s_{n-1}^{x} s_{n}^{y} s_{p}^{z}>_{0}=\frac{i}{2} \delta_{n p}<s_{n-1}^{x} s_{n}^{x}>_{0},<s_{n}^{y} s_{n+1}^{x} s_{p}^{z}>_{0}=$ $\frac{i}{2} \delta_{n p}<s_{n}^{x} s_{n+1}^{x}>_{0}$. In addition, the zero-field thermal average of a product of several operators $s^{x}$ is [16]

$$
<s_{n}^{x} s_{p}^{x}>_{0}=\frac{1}{4}\left(-\tanh \frac{J_{n}}{4 k T}\right)\left(-\tanh \frac{J_{n+1}}{4 k T}\right) \ldots\left(-\tanh \frac{J_{p-1}}{4 k T}\right), \quad n<p
$$

From eq. (11) one concludes that for $T \neq 0<s_{n}^{x} s_{n+r}^{x}>_{0} \rightarrow 0$ as $r \rightarrow \infty$ and hence $<s_{n}^{x}>_{0}=0$.

Finally, making use of eq. (11), it follows after straightforward calculations that the initial static susceptibilities (6) and (10) of the nonuniform Ising chain may be rewritten in the form

$$
\begin{aligned}
\chi_{x x}=-\frac{1}{4 k T}\left[1+\frac{2}{N} \sum_{n} \sum_{p>n}\left(-\tanh \frac{J_{n}}{4 k T}\right)\left(-\tanh \frac{J_{n+1}}{4 k T}\right) \ldots\left(-\tanh \frac{J_{p-1}}{4 k T}\right)\right] ; \\
\chi_{z z}=-\frac{1}{2 N} \sum_{n}\left[\frac{\tanh \frac{J_{n-1}-J_{n}}{4 k T}}{J_{n-1}-J_{n}}+\frac{\tanh \frac{J_{n-1}+J_{n}}{4 k T}}{J_{n-1}+J_{n}}\right. \\
\left.+\tanh \frac{J_{n-1}}{4 k T} \tanh \frac{J_{n}}{4 k T}\left(\frac{\tanh \frac{J_{n-1}+J_{n}}{4 k T}}{J_{n-1}+J_{n}}-\frac{\tanh \frac{J_{n-1}-J_{n}}{4 k T}}{J_{n-1}-J_{n}}\right)\right]
\end{aligned}
$$

The result for $\chi_{z z}(13)$ coincides with the result obtained by Idogaki, Rikitoku and Tucker [14] using a different approach.

Note that $\operatorname{Tr}\left[\mathrm{e}^{-\frac{H_{0}+H_{x}}{k T}} s_{n}^{\alpha}\right]=0, \alpha=y, z$ and therefore $\chi_{z x}=\chi_{y x}=0$. The calculation of $\operatorname{Tr}\left[\mathrm{e}^{-\frac{H_{0}+H_{z}}{k T}} s_{n}^{\alpha}\right], \alpha=x, y$ also yields 0 (after fermionization one must average a product of odd numbers of linear combinations of Fermi operators in terms of which the Hamiltonian has diagonal quadratic form) and hence $\chi_{x z}=\chi_{y z}=0$. Making use of the transformation $s^{x^{\prime}}=-s^{x}, s^{y^{\prime}}=s^{z}, s^{z^{\prime}}=s^{y}$ one concludes that $\chi_{y y}=\chi_{z z}$ and $\chi_{x y}=\chi_{z y}=0$.

It is worthwhile to note that $\chi_{z z}(13)$ does not depend on the sign of the intersite coupling, whereas $\chi_{x x}(12)$ does. For example, according to eq. (12) one obtains [16] for the regular chain $J_{1}=J_{2}=\ldots=J$ 


$$
\begin{array}{r}
\chi_{x x}=-\frac{1}{4 k T}\left[1+\frac{2}{N} \sum_{q}(N-q)\left(-\tanh \frac{J}{4 k T}\right)^{q}\right] \\
=-\frac{1}{4 k T}\left(1-\frac{2 \tanh \frac{J}{4 k T}}{1+\tanh \frac{J}{4 k T}}\right)=-\frac{\mathrm{e}^{-\frac{J}{2 k T}}}{4 k T}
\end{array}
$$

which agrees with (3). Thus one immediately finds that for $J<0$ (ferromagnetic coupling) $\chi_{x x} \rightarrow \infty$ as $T \rightarrow 0$, whereas for $J>0$ (antiferromagnetic coupling) $\chi_{x x} \rightarrow 0$ as $T \rightarrow 0$. Similarly for the case of the regular alternatingbond chain $J_{1}=J_{3}=\ldots=J_{1}, J_{2}=J_{4}=\ldots=J_{2}$, eq. (12) yields

$$
\chi_{x x}=-\frac{1}{4 k T}\left(1-\frac{\tanh \frac{J_{1}}{4 k T}+\tanh \frac{J_{2}}{4 k T}-2 \tanh \frac{J_{1}}{4 k T} \tanh \frac{J_{2}}{4 k T}}{1-\tanh \frac{J_{1}}{4 k T} \tanh \frac{J_{2}}{4 k T}}\right)
$$

As can be seen from eq. (15) and figs. 1-4 (the dashed curves) for $J_{1}, J_{2}<0 \chi_{x x}$ diverges with $T \rightarrow 0$, whereas for $J_{1}<0, J_{2}>0, \quad J_{1}>0, J_{2}<0, \quad J_{1}, J_{2}>0 \chi_{x x}$ does not diverge with $T \rightarrow 0$.

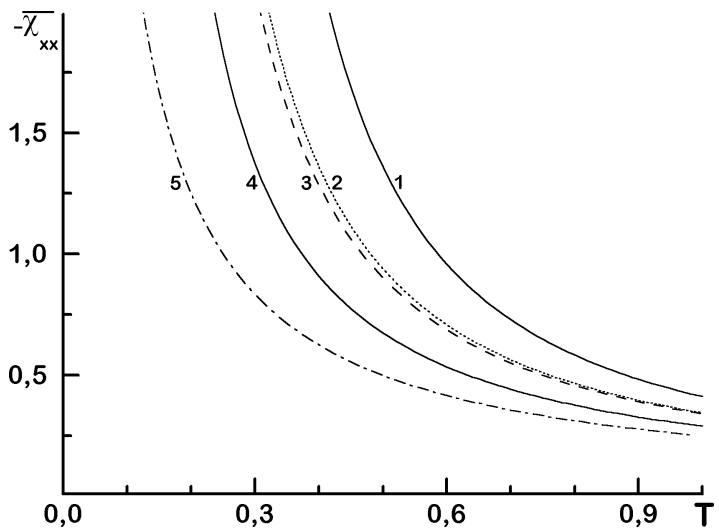

Fig. 1. The temperature dependence of the random-averaged initial longitudinal static susceptibility for the disorder given by (18) with $J_{1}=-1, J_{2}=-0.3 ; 1-c=1$, $2-c=0.5,3-$ regular alternating-bond chain, $4-c=0$, $5-J_{1}=J_{2}=0$ (ideal paramagnetic).

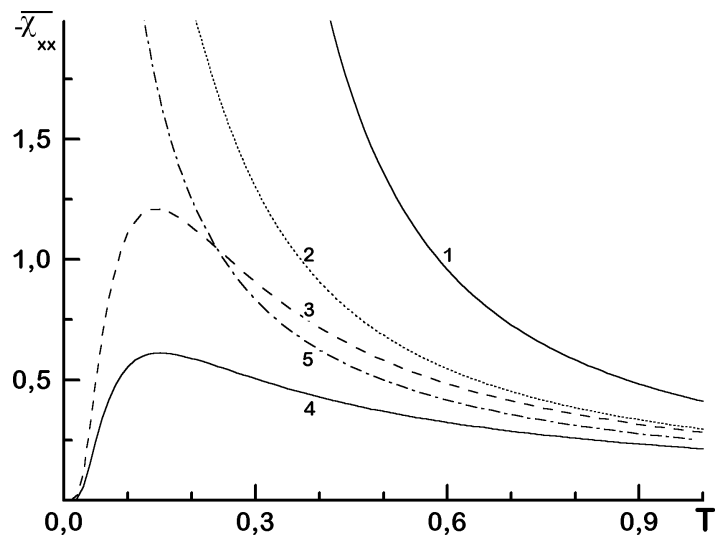

Fig. 2. The same as in fig. 1 for $J_{1}=-1, J_{2}=0.3$.

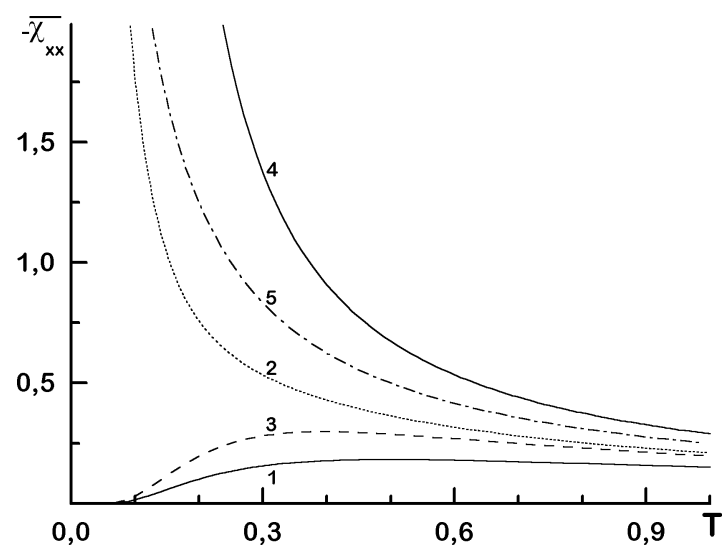

Fig. 3. The same as in fig. 1 for $J_{1}=1, J_{2}=-0.3$.

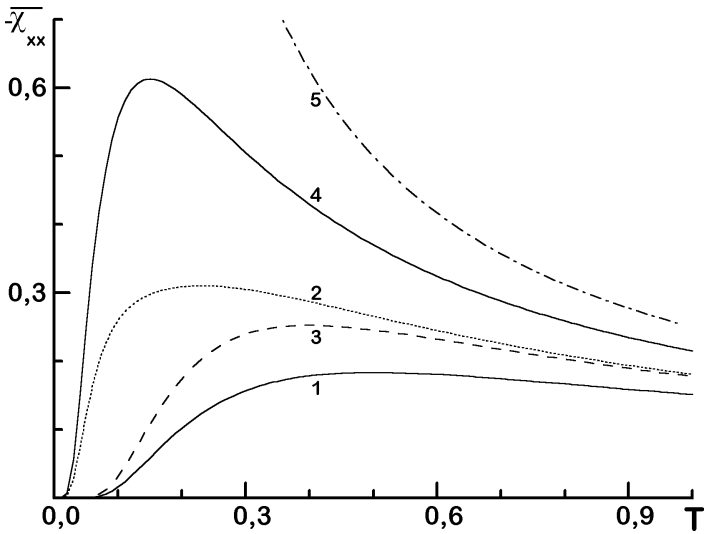

Fig. 4. The same as in fig. 1 for $J_{1}=1, J_{2}=0.3$. 


\section{INITIAL STATIC SUSCEPTIBILITIES OF A RANDOM ISING CHAIN}

We now specialize the theory of nonuniform chain to a study of random chain (1) assuming the exchange couplings to be random variables evenly distributed with the probability density $p\left(J_{n}\right)$. The random averaged susceptibilities are given by

$$
\begin{aligned}
\overline{\chi_{x x}} & =-\frac{1}{4 k T}\left[1+\frac{2}{N} \sum_{q}(N-q){\left.\overline{\left(-\tanh \frac{J}{4 k T}\right)^{q}}\right]}^{q} \overline{\frac{2 \tanh \frac{J}{4 k T}}{4 k T}}\left(1-\frac{1}{1+\overline{\tanh \frac{J}{4 k T}}}\right)\right.
\end{aligned}
$$

(in the first equality we have made use of the fact that $\overline{-\tanh \frac{J_{p}}{4 k T}}$ is the same for all sites $p$ ), and

$$
\begin{aligned}
\overline{\chi_{z z}}= & -\frac{1}{2} \overline{\left(\frac{\tanh \frac{J_{1}-J_{2}}{4 k T}}{J_{1}-J_{2}}\right)}+\overline{\left(\frac{\tanh \frac{J_{1}+J_{2}}{4 k T}}{J_{1}+J_{2}}\right)} \\
& \left.+\overline{\left(\tanh \frac{J_{1}}{4 k T} \tanh \frac{J_{2}}{4 k T} \frac{\tanh \frac{J_{1}+J_{2}}{4 k T}}{J_{1}+J_{2}}\right)}-\overline{\left(\tanh \frac{J_{1}}{4 k T} \tanh \frac{J_{2}}{4 k T} \frac{\tanh \frac{J_{1}-J_{2}}{4 k T}}{J_{1}-J_{2}}\right)}\right]
\end{aligned}
$$

$\underline{\text { Here }}$ the random average is defined by the relation $\overline{(\ldots)} \equiv \ldots \int d J_{n} p\left(J_{n}\right) \ldots(\ldots)$. Besides the probability distribution

$$
\begin{aligned}
& p\left(J_{n}\right)=c \delta\left(J_{n}-J_{1}\right)+(1-c) \delta\left(J_{n}-J_{2}\right), \\
& 0 \leq c \leq 1
\end{aligned}
$$

for which $\overline{\chi_{z z}}$ was examined in ref. [14] we shall consider the Gaussian distribution

$$
p\left(J_{n}\right)=\frac{1}{\sqrt{2 \pi} \sigma} \mathrm{e}^{-\frac{\left(J_{n}-J_{0}\right)^{2}}{2 \sigma^{2}}}
$$

and the Lorentzian distribution

$$
p\left(J_{n}\right)=\frac{1}{\pi} \frac{\Gamma}{\left(J_{n}-J_{0}\right)^{2}+\Gamma^{2}}
$$

centred at $J_{0}$ with the strength of disorder controlled by $\sigma^{2}$ and $\Gamma$, respectively.

Let us consider at first the longitudinal susceptibility $\overline{\chi_{x x}}$ (16). Its low-temperature behaviour for nonrandom case can be derived from the second equality in (14) after taking into account that $\tanh \frac{J}{4 k T} \rightarrow \operatorname{sgn} J$ while $T \rightarrow 0$. As a result $\chi_{x x}$ diverges more rapidly than $\frac{1}{T}\left(\frac{1}{T} \cdot \infty\right)$ while $T \rightarrow 0$ for $J<0$, and does not diverge $\left(\frac{1}{T} \cdot 0\right)$ while $T \rightarrow 0$ for $J>0$ (compare solid curves in figs. 1-6). After introducing any small amount of randomness for which $\left|\overline{\tanh \frac{J}{4 k T}}\right|<1$ (for example, disorder (18) with different signs of $J_{1}$ and $J_{2}$ or the disorders defined by (19), (20)) the temperature dependence for $T \rightarrow 0$ changes drastically $\left(\frac{1}{T} \cdot\right.$ const) and becomes similar to the ideal paramagnetic (the dotted curves in figs. 2, 3, and 5,6$)$. Thus, the introducing of such types of randomness leads to qualitative changes in the dependence $\overline{\chi_{x x}}$ against $T$. However, if after the introducing of disorder $\left|\overline{\tanh \frac{J}{4 k T}}\right|$ still equals to 1 while $T \rightarrow 0$ (the disorder (18) with the same signs of $J_{1}$ and $J_{2}$ ) the introducing of disorder leads to the quantitative changes rather than to the qualitative ones (the dotted curves in figs. 1, 4).

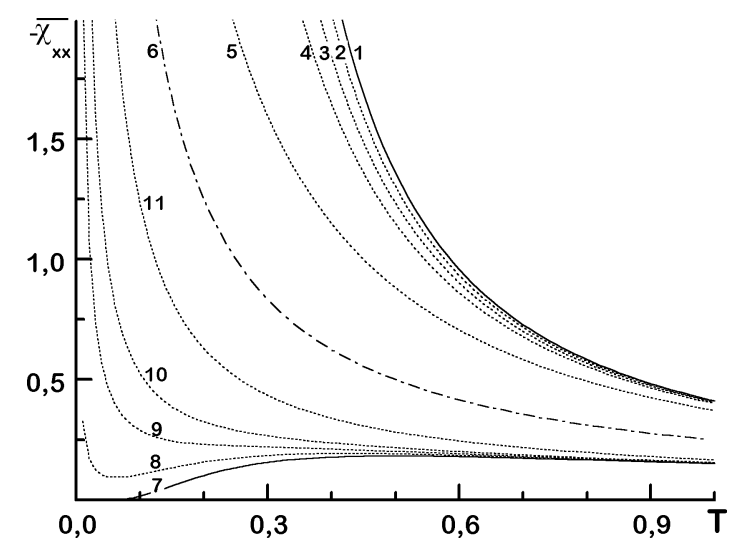

Fig. 5. The temperature dependence of the random-averaged initial longitudinal static susceptibility for the disorder given by (19); $J_{0}=-1: 1-\sigma^{2}=0,2-\sigma^{2}=0.2,3-$ $\sigma^{2}=0.5,4-\sigma^{2}=1,5-\sigma^{2}=5 ; 6-J_{0}=\sigma^{2}=0$ (ideal paramagnetic); $J_{0}=1: 7-\sigma^{2}=0,8-\sigma^{2}=0.2,9-$ $\sigma^{2}=0.5,10-\sigma^{2}=1,11-\sigma^{2}=5$. 


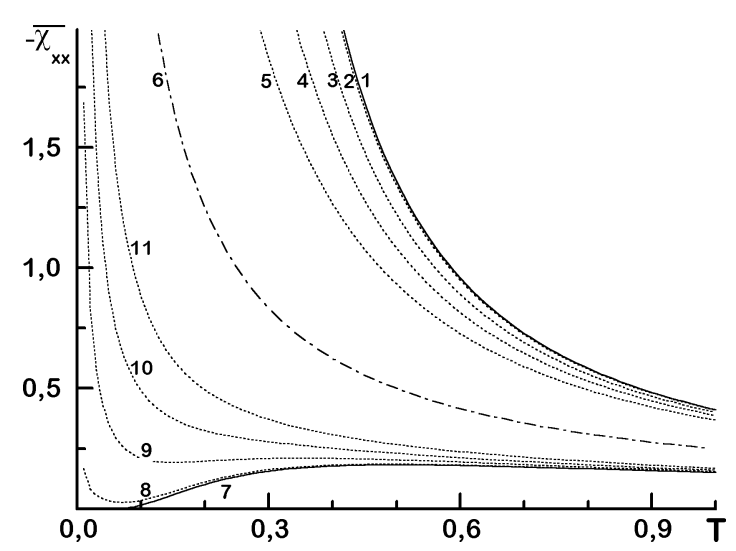

Fig. 6. The temperature dependence of the random-averaged initial longitudinal static susceptibility for the disorder given by $(20) ; J_{0}=-1: 1-\Gamma=0,2-\Gamma=0.02$, $3-\Gamma=0.2,4-\Gamma=0.5,5-\Gamma=1 ; 6-J_{0}=\Gamma=0$ (ideal paramagnetic); $J_{0}=1: 7-\Gamma=0,8-\Gamma=0.02,9-$ $\Gamma=0.2,10-\Gamma=0.5,11-\Gamma=1$.

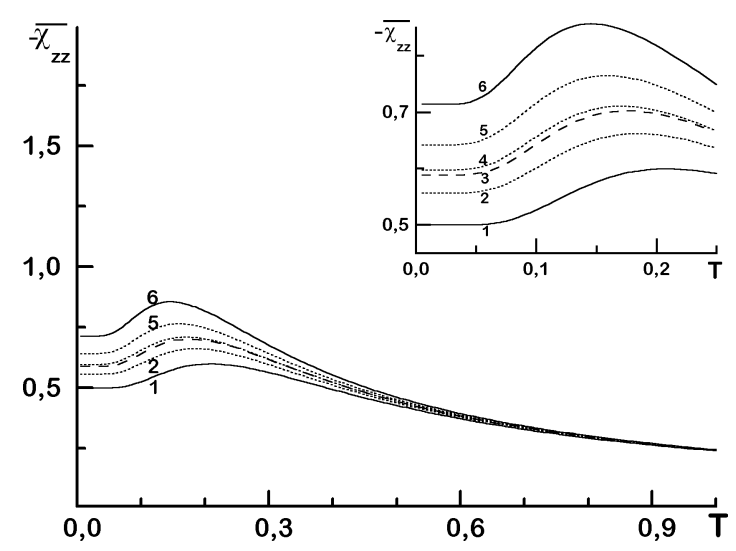

Fig. 7. The temperature dependence of the random-averaged initial transverse static susceptibility for the disorder given by (18) with $J_{1}=1, J_{2}=0.7 ; 1-c=1,2-$ $c=0.7,3$ - regular alternating-bond chain, $4-c=0.5,5$ $-c=0.3,6-c=0$.

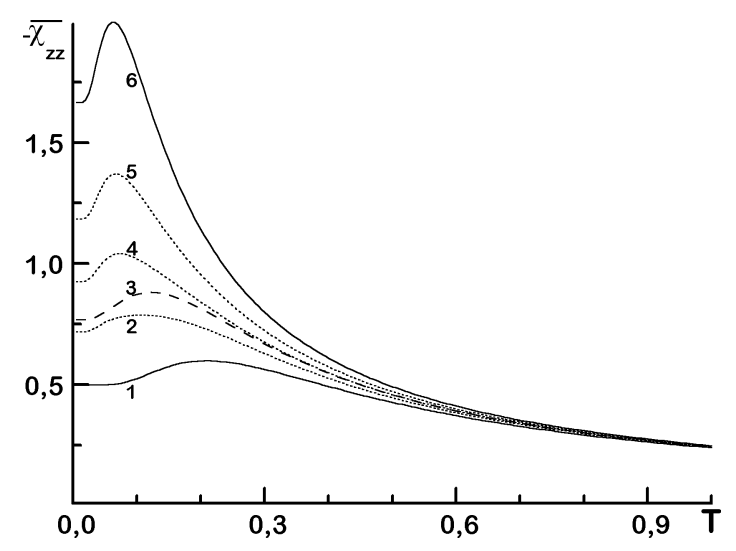

Fig. 8. The same as in fig. 7 for $J_{1}=1, J_{2}=0.3$.

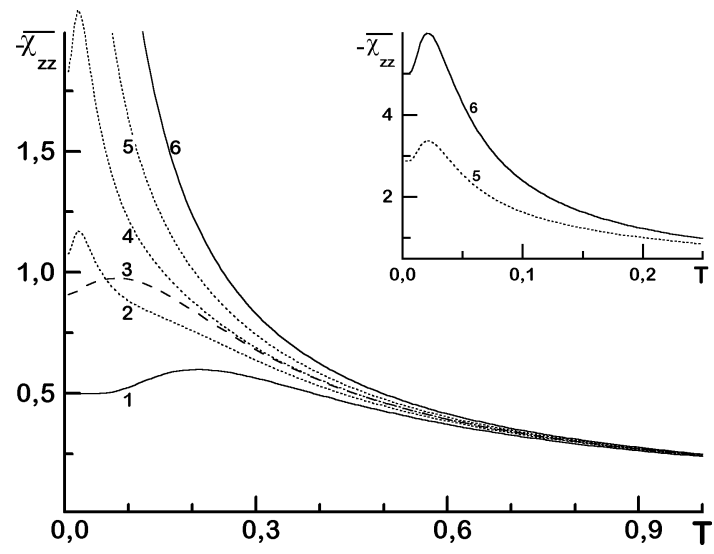

Fig. 9. The same as in fig. 7 for $J_{1}=1, J_{2}=0.1$.

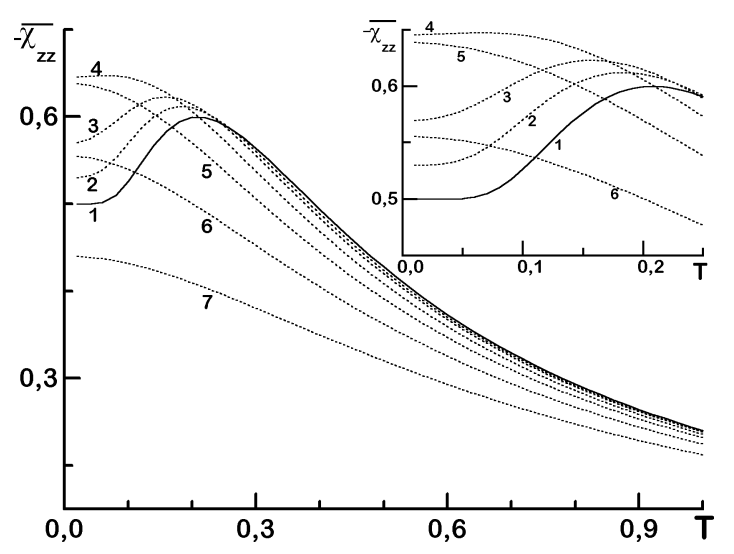

Fig. 10. The temperature dependence of the random-averaged initial transverse static susceptibility for the disorder given by (19) with $J_{0}=1 ; 1-\sigma^{2}=0,2-\sigma^{2}=0.1$, $3-\sigma^{2}=0.2,4-\sigma^{2}=0.5,5-\sigma^{2}=1,6-\sigma^{2}=2,7-$ $\sigma^{2}=4$.

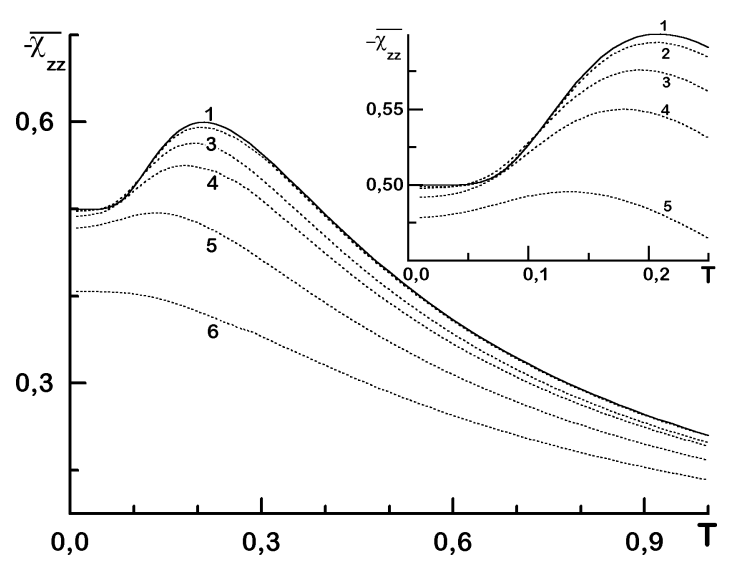

Fig. 11. The temperature dependence of the random-averaged initial transverse static susceptibility for the disorder given by (20) with $J_{0}=1 ; 1-\Gamma=0,2-\Gamma=0.02$, $3-\Gamma=0.1,4-\Gamma=0.2,5-\Gamma=0.5,6-\Gamma=1$. 
Another observation concerns regular alternatingbond chains and random chains with the disorder given by (18). As it has been noted already in connection with eq. (15) $\chi_{x x}$ diverges as $T \rightarrow 0$ for alternatingbond chain with $J_{1}, J_{2}<0$ and does not diverge if $J_{1}<0, J_{2}>0, \quad J_{1}>0, J_{2}<0$ or $J_{1}, J_{2}>0$ (the dashed curves in figs. 1-4). The random-bond chain with two types of exchange bonds $J_{1}$ and $J_{2}$ that appear with the same probability may exhibit essentially different temperature behaviour of $\overline{\chi_{x x}}$. Namely, for the cases $J_{1}<0, J_{2}>0$ and $J_{1}>0, J_{2}<0 \overline{\chi_{x x}}$ diverges with $T \rightarrow 0$ as $\frac{1}{T}$ (see eq. (16)) and the dotted curves in figs. 2,3 . However, as can be seen from eqs. (15) and (16) for the cases $J_{1}, J_{2}<0, J_{1}, J_{2}>0$ the difference between alternating-bond and random-bond chains is only quantitative (the dotted curves in figs. 1,4).

Let us discuss the temperature behaviour of the initial transverse static susceptibility $-\overline{\chi_{z z}}(17)$. For the nonrandom case it is finite at all temperatures, equals to $\frac{1}{2 J}$ at $T=0$, slightly increases with increase of the temperature and then decreases approaching the temperature dependence for the ideal paramagnetic at high temperatures. The weaker is the exchange interaction between $x$ spin components the larger is the value of $-\chi_{z z}$ at $T=0$, the smaller is the temperature at which $-\chi_{z z}$ exhibits maximum, the smaller are the temperatures at which $-\chi_{z z}$ exhibits the behaviour inherent in the ideal paramagnetic (see the solid curves in figs. 7-11). Figs. $7-9$ show the rebuilding of the temperature dependence of $\overline{\chi_{z z}}$ for disorder (18) from the case $J_{2}=0.7,0.3,0.1$ to $J_{1}=1$ with the increasing of $c$ from 0 to 1 . We also depicted the susceptibilities of the corresponding regular alternating-bond chains. It can be seen that the model with randomness exhibits an enhancement of $\overline{\chi_{z z}}$ at low temperatures (compare the dotted and the dashed curves in figs. 7-9), and therefore, one may say that the effective exchange coupling is weakened by the randomness.

Somewhat different is the influence of the disorders given by (19) and (20) on the temperature dependence of $\overline{\chi_{z z}}$ (figs. 10, 11). For the Gaussian distribution with small strength of disorder one observes an enhancement of $-\overline{\chi_{z z}}$ at low temperatures (the curves $2-6$ in fig. 10). A further increasing of the disorder leads to the suppression of low-temperature values of $-\overline{\chi_{z z}}$ (the curve 7 in fig. 10). In contrast to the Gaussian disorder the Lorentzian disorder leads to decrease of $-\overline{\chi_{z z}}$ almost at all temperatures (the dotted curves in fig. 11). Such a behaviour apparently is conditioned by long tails of Lorentzian distribution (20) that suppose large intersite couplings yielding a suppression of the initial transverse static susceptibility.

\section{DISCUSSION}

Let us summarize. We presented some exact calculations for the initial static susceptibilities of the uniform and random spin- $\frac{1}{2}$ Ising chains. Considering several types of randomness we found that the disorder may lead either to quantitative or to qualitative changes in the temperature dependence of the initial static susceptibilities. In particular, depending on the type of disorder we observed either a quantitative or a qualitative difference between the initial longitudinal static susceptibilities of the regular alternating-bond and random-bond chains with two types of exchange bonds. We revealed the difference in the influence of the Gaussian and Lorentzian disorders on the temperature dependence of the initial transverse static susceptibility. We hope that the derived conclusions will be useful for understanding the effects of disorder on observable properties of the corresponding quasi-one-dimensional compounds.

\section{ACKNOWLEDGEMENTS}

The authors are grateful to Professor J. W. Tucker for useful correspondence and critical reading of the manuscript. The paper was presented in part at the Ising Lectures '97 (ICMP, Lviv). The authors thank the participants for discussion.
[1] E. Izing, Z. Phys. 31, 253 (1925) (in German).

[2] R. J. Baxter F. R. S., Exactly Solved Models in Statistical Mechanics (Academic Press: London New York Paris San Diego San Francisco São Paulo Sydney Tokyo Toronto, 1982).

[3] P. Pfeuty, Ann. Phys. (N. Y.) 57, 79 (1970).

[4] B. K. Chakrabarti, A. Dutta, P. Sen, Quantum Ising Phases and Transitions in Transverse Ising Models (Springer: Berlin Heidelberg, 1996).

[5] G. Müller, R. E. Shrock, Phys. Rev. B 30, 5254 (1984).

[6] G. Müller, R. E. Shrock, Phys. Rev. B 31, 637 (1985).

[7] C. Fan, B. M. McCoy, Phys. Rev. 182, 614 (1969).

[8] E. Barouch, B. M. McCoy, Stud. Appl. Math. 51, 57 (1972).
[9] R. O. Zaitsev, Zh. Eksp. Teor. Fiz. 63, 1487 (1972).

[10] B. Ya. Balagurov, V. G. Vaks, R. O. Zaitsev, Fiz. Tverd. Tela 16, 2302 (1974).

[11] H. Braeter, J. W. Kowalski, Physica A 87, 243 (1977).

[12] O. Derzhko, T. Krokhmalskii, T. Verkholyak, Mater. Sci. Eng. A 226-228, 1049 (1997).

[13] F. Iglói, J. Phys. A: Math. Gen. 27, 2995 (1994).

[14] T. Idogaki, M. Rikitoku, J. W. Tucker, J. Magn. Magn. Mater. 152, 311 (1996).

[15] D. N. Zubarev, Nieravnoviesnaia Statistichieskaia Tiermodinamika (Nonequilibrium Statistical Thermodynamics) (Nauka, Moscow, 1971).

[16] H. E. Stanley, Introduction to phase transitions and critical phenomena (Clarendon Press: Oxford, 1971). 


\section{СТАТИЧНІ СПРИЙНЯТЛИВОСТІ НЕОДНОРІДНИХ І ВИПАДКОВИХ ЛАНЦЮЖКІВ ІЗИНГА}

\section{О. Держко, О. Забуранний}

Інститут фізики конденсованих систем НАН Украӥни,

Україна, UА-290011, Лъвів, вул. Свєниічвкого, 1

Лъвівсъкий державний університет імені Івана Франка,

Україна, UA-290005, Лъвів, вул. Драгоманова, 12

Для неоднорідного спін- $\frac{1}{2}$ ланцюжка Ізинга з гамільтоніяном

$$
H_{0}=\sum_{n} J_{n} s_{n}^{x} s_{n+1}^{x}
$$

ми отримали точні вирази для початкових (при нульовому полі) сприйнятливостей:

$$
\begin{aligned}
\chi_{x x} & =-\frac{1}{4 k T}\left[1+\frac{2}{N} \sum_{n} \sum_{p>n}\left(-\tanh \frac{J_{n}}{4 k T}\right)\left(-\tanh \frac{J_{n+1}}{4 k T}\right) \ldots\left(-\tanh \frac{J_{p-1}}{4 k T}\right)\right], \\
\chi_{y y} & =\chi_{z z}=-\frac{1}{2 N} \sum_{n}\left[\frac{\tanh \frac{J_{n-1}-J_{n}}{4 k T}}{J_{n-1}-J_{n}}+\frac{\tanh \frac{J_{n-1}+J_{n}}{4 k T}}{J_{n-1}+J_{n}}\right. \\
& \left.+\tanh \frac{J_{n-1}}{4 k T} \tanh \frac{J_{n}}{4 k T}\left(\frac{\tanh \frac{J_{n-1}+J_{n}}{4 k T}}{J_{n-1}+J_{n}}-\frac{\tanh \frac{J_{n-1}-J_{n}}{4 k T}}{J_{n-1}-J_{n}}\right)\right],
\end{aligned}
$$

$\chi_{\alpha \beta}=0, \alpha \neq \beta$. Ці результати дають змогу вивчати ланцюжки Ізинга з випадковими обмінними взаємодіями $J_{n}$. Ми розглянули кілька щільностей розподілу ймовірности $p\left(J_{n}\right)$ і дослідили вплив внесеного безладу на температурну залежність початкових статичних сприйнятливостей. 